\title{
Polydimethylsiloxane as a Matrix for the Stabilization and Immobilization of Zero-Valent Iron Nanoparticles. Applications to Dehalogenation of Environmentally Deleterious Molecules
}

\author{
Donaldo Fabio Mercado ${ }^{a}$ and Richard G. Weiss ${ }^{*, a, b}$ \\ ${ }^{a}$ Department of Chemistry, Georgetown University, 20057-1227 Washington, DC, USA \\ ${ }^{b}$ Institute for Soft Matter Synthesis and Metrology, Georgetown University, \\ 20057-1227 Washington, DC, USA
}

\begin{abstract}
A wet-synthetic method is described to obtain magnetic, air-stable, zero-valent iron nanoparticles coated with an alkanedioic acid. The particles can be immobilized in an amino-substituted polysiloxane matrix to provide materials capable of dechlorinating, debrominating, and deiodinating a wide variety of halogenated organic molecules dissolved initially in a highly polar medium. The methodology involves diffusion of the organic molecules into the polysiloxane matrix, their reaction with the embedded iron nanoparticles, and then diffusion of the dehalogenated products back into the polar medium or into the air. Thus, the abilities of the coated particles, as suspensions or within an amino-substituted polysiloxane matrix crosslinked with succinic acid, to dehalogenate various organic molecules (especially the model compounds, bromobenzene and chlorobenzene), have been assessed. Data from several spectroscopic methods demonstrate that the particles are in a crystalline $\alpha$-Fe phase surrounded by the diacid shell. The effect of embedding $10 \mathrm{wt} . \%$ of the particles in amino-substituted polysiloxane-diacid matrices with different $\mathrm{COOH} / \mathrm{NH}_{2}$ group ratios and the viscoelastic properties of the polymeric materials have been investigated as well. Potential uses of these gel-like materials to remove halogenated pollutants from various types of aquifers are mentioned.
\end{abstract}

Keywords: zero-valent iron nanoparticles, amino-substituted polydimethylsiloxane, absorption, dehalogenation

\section{Introduction}

We describe a wet method for synthesizing and stabilizing zero-valent iron nanoparticles $\left(\mathrm{nFe}^{0}\right)$ toward atmospheric oxygen and water and then their introduction into a polymeric matrix. The matrix assures immobilization of the particles while allowing their easy recovery after use to dehalogenate a variety of environmentally undesirable organic molecules. A key aspect of this approach includes the selection of the polymer matrix, an amino-substituted polysiloxane. It interacts strongly with $\mathrm{nFe}^{0}$ particles, which are covered by a protective layer of dicarboxylic acid molecules that excludes water while allowing the entry of halogenated molecules that are absorbed into the polymer and then diffuse to the $\mathrm{nFe}^{0}$ particles to react. This approach will allow the $\mathrm{nFe}^{0}$ species to be placed in aqueous and aerated locations for long periods, where they can perform the desired dehalogenations and then be

*e-mail: weissr@georgetown.edu recovered at will. The experimental results presented here, both in the absence and presence of the polymer matrix, demonstrate the feasibility of our approach in a laboratory setting and establish the feasility of the materials to act in future experiments to remove pollutants from lakes, streams and underground water supplies.

Halogenated organic compounds (HOCs) are widely used as solvents, pesticides, flame-retardants, herbicides, disinfectants, and wood preservatives. ${ }^{1,2}$ Because they are relatively stable and tend to accumulate in the environment without adequate treatment after their use, they tend to enter the water sources and soil, ${ }^{3}$ where they can be ingested by and accumulate in animals. ${ }^{4,5}$ In addition, in situ formation of some HOCs and contamination of water sources occurs when chlorine is used for disinfection proposes and reacts with humic and fulvic substances ${ }^{6,7}$ HOCs in the environment are a risk to the health of humans and other animals, many are toxic, carcinogenic, and exhibit mutagenic activity. ${ }^{1,8}$ As a result, interest in developing processes, materials and technologies for remediation 
of HOCs is growing. Adsorption, ${ }^{9}$ phytoremediation, ${ }^{10}$ bioprocessing ${ }^{11}$ and chemical oxidation/reduction systems $\mathrm{s}^{12,13}$ are among the techniques being employed in this regard. Also, some nanotechnologies for water treatment are promising as efficient 'remediators' because of the high surface/volume ratios intrinsic to nanomaterials. ${ }^{14}$

In that regard, zero-valent iron nanoparticles $\left(\mathrm{nFe}^{0}\right)$ are useful for the dehalogenation of HOCs $;{ }^{15-17}$ they are highly reactive toward halogenated molecules and low in cost. ${ }^{14,18}$ However, they react rapidly as well with oxygen in the air or dissolved in a liquid, ${ }^{19}$ leading to iron oxides which are less efficient as dehalogenation agents. For that reason, these materials typically have an iron-zero phase in the core and an iron-oxide phases on the surface. ${ }^{20,21}$ Approaches for obtaining air-stable $\mathrm{nFe}^{0}$ require the development of methods to control their surface chemistry. In addition, an advantage of using $\mathrm{nFe}^{0}$ in situ for water purification is that their high magnetic response ${ }^{22}$ can facilitate their removal after an aquifer has been treated. However, without appropriate surface treatment, those magnetic properties can lead to tight agglomeration of the particles, ${ }^{23,24}$ thereby diminishing their effective surface-to-volume ratios and, thus, efficiency for removal of pollutants. ${ }^{25}$ Although water currents may disperse the particles, hampering their recovery, there is little information about the toxicity or risks associated with leaving $\mathrm{nFe}^{0}$ in the environment. ${ }^{26}$ Thus, methods to produce air-stable $\mathrm{nFe}^{0}$ and to deliver them in formulations that maintain their activity without compromising their retrieval are needed.

Our approach to resolve these issues is two-fold: (i) place a coating of an alkanedioic acid on the surface of the nanoparticles to make them stable in air and water; (ii) immobilize the coated particles in a polymeric matrix that keeps them from being dispersed over large volumes but allows the halogenated molecules access to them. The polymer matrix selected is one of several commercially available amino-substituted polydimethylsiloxanes, $\mathrm{NH}_{2}$ PDMS.

Polydimethylsiloxane (PDMS) is one of the most studied and common polymers. ${ }^{27}$ It consists of $\left[-\mathrm{Si}(\mathrm{Me})_{2}-\mathrm{O}-\right]_{\mathrm{n}}$ chains. Changing the $-\mathrm{Si}-\mathrm{O}-$ chain length or the nature of the side groups has resulted in a family of materials with a wide range of properties. Amino-substitution on side chain groups in $\mathrm{NH}_{2} \mathrm{PDMS}$ allows the polymer chains to interact specifically with a variety of functional groups which, in turn, produce materials whose microscopic and bulk properties can be very different from those of the parent PDMS. In that regard, it has been shown that the $\mathrm{NH}_{2} \mathrm{PDMS}$ are able to create ion pairs with the triatomic molecules, $\mathrm{CO}_{2}$ and $\mathrm{CS}_{2},{ }^{27}$ and with mono- and di-carboxylic acids ${ }^{28}$ to produce crosslinks in which the resulting materials have a variety of attractive swelling, rheological, and adhesive properties. A generic $\mathrm{NH}_{2} \mathrm{PDMS}$ containing monomers with 3-aminopropyl side chains and an alkanedioic acid (mDCA, where $\mathrm{m}$ is the number of carbon atoms in the chain) react to form ionic crosslink through formation of carboxylate anions and ammonium cations. ${ }^{28}$

Only $\mathrm{NH}_{2} \mathrm{PDMS}$ with 6-7\% of the monomers containing an amino group has been used in this study. Other copolymers with different percentages of 3-aminopropryl and other amino side chains are available commercially. Clearly, they can be employed to produce materials with macroscopic properties and dehalogenation efficiencies different from the one reported here. In addition, mDCAs are able to chelate $\mathrm{Fe}^{+2}$ and $\mathrm{Fe}^{+3}$ in solution, ${ }^{16}$ and the $\mathrm{nFe}^{0}$ coated with an mDCA are stable in the air, under water, or embedded in $\mathrm{NH}_{2} \mathrm{PDMS}$ matrices for several months. The period of stability of these $\mathrm{nFe}^{0}$ particles in the air is longer than has been reported with other coatings of which we are aware. ${ }^{29}$

Here, we report the syntheses of $\mathrm{nFe}^{0}$-mDCA particles, especially adipic acid (i.e., $\mathrm{m}=6$ ), as the surface stabilizers. Also, the materials with 6DCA were made at 0,25 and $60{ }^{\circ} \mathrm{C}$. The particles are designated $\mathrm{nFe}^{0}-\mathrm{mDCA}-{ }^{\circ} \mathrm{C}$; for example, the one made with adipic acid at $60{ }^{\circ} \mathrm{C}$ is denoted $n \mathrm{ne}^{0}-6 \mathrm{DCA}-60$. Note that all of the particles were synthesized at $25^{\circ} \mathrm{C}$ except $6 \mathrm{DCA}$ as indicated. The mDCA coating protects the interior iron atoms from additional oxidation while permitting continued activity by the $\mathrm{nFe}^{0}$ as dehalogenating agents. Their reactivity as dehalogenating agents has been demonstrated when they were placed in a polar solvent, methanol, or when incorporated within $\mathrm{NH}_{2}$ PDMS-4DCA composites. The composites result from quaternization reactions of amino groups between 4DCA molecules as well as from some free $-\mathrm{COOH}$ groups on the $\mathrm{nFe}^{0}$-mDCA surface (Scheme 1).

The dehalogenation results from experiments with several halogenated model compounds, especially chlorobenzene and bromobenzene, are reported using $\mathrm{nFe}^{0}$ made by different protocols and with different mDCAs as suspensions or embedded in $\mathrm{NH}_{2} \mathrm{PDMS}$ 4DCA matrices at different $\mathrm{COOH} / \mathrm{NH}_{2}$ group ratios. Results from experiments with other HOCs (n.b., iodobenzene, fluorobenzene, 1-chloro-3-fluorobenzene, 2-fluoro-4-methylpyridine, 4-fluorobenzonitrile, methyl bromoacetate, ethyl iodoacetate, chloroacetic acid, dichloroacetic acid, trichlorooacetic acid and trichloroethylene) are presented, as well.

In total, they demonstrate the limits of the dehalogenation processes, and that a very broad range of $\mathrm{nFe}^{0}$ based materials can be made air-stable easily and 'tuned' for specific applications. 


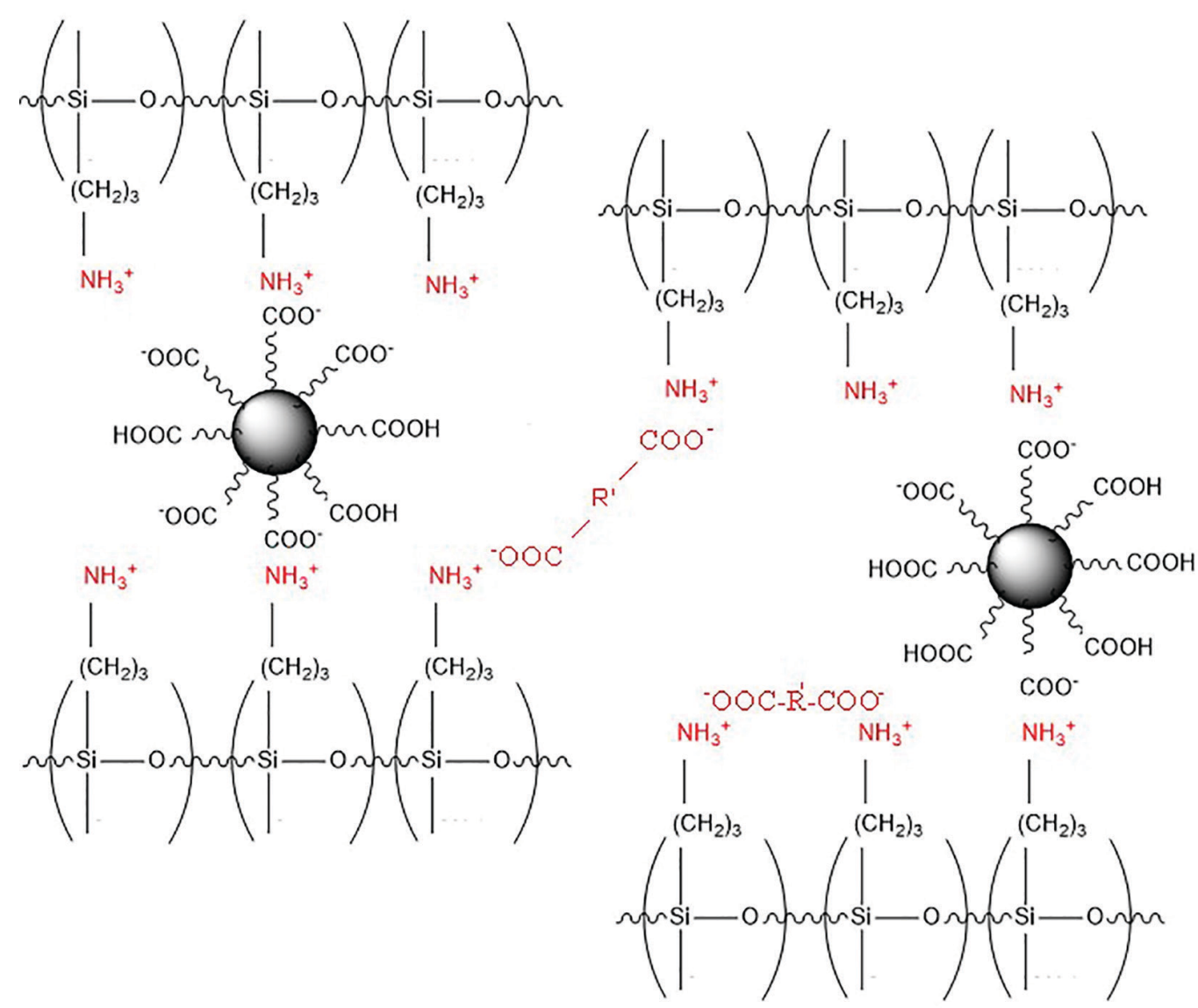

Scheme 1. Representative structures of ionic pairs produced upon reaction between $\mathrm{nFe} \mathrm{e}^{0}$-mDCA and an $\mathrm{NH}_{2} \mathrm{PDMS}_{\text {to }}$ which an mDCA (which adopts its dianionic form, -OOC-R'-COO-) has been added.

\section{Experimental}

\section{Reactants}

Ferrous sulfate heptahydrate $\left(\mathrm{FeSO}_{4} \cdot 7 \mathrm{H}_{2} \mathrm{O}, 98 \%\right)$ and sulfuric acid $\left(\mathrm{H}_{2} \mathrm{SO}_{4}, 95.2 \%\right)$ were obtained from Fisher. Sodium borohydride $\left(\mathrm{NaBH}_{4}, 98 \%\right)$, sodium hydroxide (NaOH, 98\%), methanol (99\%), hexane (99\%), all mDCAs (oxalic, succinic, adipic, suberic, sebacic and dodecanedioic acids, 99\%), chloroacetic acid (99\%), dichloroacetic acid $(99 \%)$, trichloroacetic acid (99\%), 2-fluoro4-methylpyridine (98\%) and 4-fluorobenzonitrile (99\%) were obtained from Sigma-Aldrich. Bromobenzene (99\%), chlorobenzene (99\%), fluorobenzene (99\%), iodobenzene (99\%), 1-chloro-3-fluorobenzene (99\%), benzene (99\%), methyl bromoacetate $(97 \%)$, ethyl iodoacetate $(98 \%)$, methyl-acetate $(99 \%)$ and trichloroethylene (TCE, 99.5\%) were supplied by Alfa Aesar. (3-Aminopropyl)methylsiloxane-co-dimethysiloxane copolymer with (6-7) wt.\% monomer units with amino groups $\mathrm{NH}_{2} \mathrm{PDMS}$, Mw (molecular weight) 4000-5000, viscosity 0.08-0.12 Pa s (characterization data from supplier) was from Gelest. All reactants were used as received.

\section{Synthesis of $\mathrm{nFe}^{0}$ nanoparticles ${ }^{30}$}

In a typical synthesis, $3 \mathrm{~g}$ of $\mathrm{FeSO}_{4} \cdot 7 \mathrm{H}_{2} \mathrm{O}$ were dissolved in $200 \mathrm{~mL}$ of deionized water at $25^{\circ} \mathrm{C}$ (unless noted otherwise). $1 \mathrm{~mL}$ of concentrated $\mathrm{H}_{2} \mathrm{SO}_{4}$ was added. The resulting solution was purged with $\mathrm{N}_{2}$ and it was stirred at $1150 \mathrm{rpm}$ for $30 \mathrm{~min}$ at the desired temperature (vide infra). Separately, a corresponding amount of $\mathrm{mDCA}$ was suspended in $200 \mathrm{~mL}$ of $\mathrm{H}_{2} \mathrm{O}$, and $3 \mathrm{~mL}$ of $5 \mathrm{M} \mathrm{NaOH}$ were added to dissolve it. The solution of mDCA was added dropwise to the $\mathrm{FeSO}_{4}$ solution. The functional group ratio of $\mathrm{Fe}:-\mathrm{COOH}$ was 1:4 in all cases. Also, the $\mathrm{pH}$ of the mixture was adjusted to 6.5 with aqueous $\mathrm{NaOH}$. Immediately thereafter, $200 \mathrm{~mL}$ of a freshly prepared $2 \mathrm{M}$ $\mathrm{NaBH}_{4}$ aqueous solution was added dropwise. The system was stirred while bubbling $\mathrm{N}_{2}$ through the liquid for $30 \mathrm{~min}$ after the completion of the addition of $\mathrm{NaBH}_{4}$. A black emulsion was present at this point. $200 \mathrm{~mL}$ of methanol were added and the mixture was stirred at $750 \mathrm{rpm}$ during 5 min. The black magnetic particles were separated from the liquid using a magnet and they were washed three times with deoxygenated water and one time with deoxygenated ethanol. The black solid was dried at $60{ }^{\circ} \mathrm{C}$ under vacuum 
( 25 torr) for $24 \mathrm{~h}$. During the whole process the reaction mixture was stirred and nitrogen was bubbled through the liquid.

\section{Synthesis of the polymeric matrix}

Only succinic acid (4DCA) was used as the crosslinker to obtain the polymeric matrix with the $\mathrm{NH}_{2} \mathrm{PDMS}$. $2 \mathrm{~g} \mathrm{NH}_{2}$ PDMS were added to an amount of 4DCA (100, 70,50 or $20 \mathrm{mg}$, corresponding to $100,70,50$ and $20 \%$ carboxyl/amino functional group ratios, respectively), that had been dissolved in $20 \mathrm{~mL}$ of $1: 1$ (v:v) acetone:pentane. After stirring the mixture at room temperature for $48 \mathrm{~h}$, the solvents were removed on a rotatory evaporator at $60{ }^{\circ} \mathrm{C}$. The resulting soft material is designated $\mathrm{NH}_{2} \mathrm{PDMS}$-ratio, where the amount of 4DCA added is indicated by the percent of acid groups to amino groups; for example, the polymer with 0.7 carboxylic groups to 1.0 amine groups is $\mathrm{NH}_{2} \mathrm{PDMS}-70$.

\section{Synthesis of the polymeric matrix with $\mathrm{nFe}^{0}$ particles}

The procedure was the same as mentioned for preparation of the polymeric matrix without particles except that the desired amount of 4DCA was added to $20 \mathrm{~mL}$ of a solution containing $2.0 \mathrm{~g}$ of $\mathrm{NH}_{2}$ PDMS in $1: 1(\mathrm{v}: \mathrm{v})$ acetone:pentane and stirred for $20 \mathrm{~min}$ before adding $200 \mathrm{mg}$ of $\mathrm{nFe}^{0}-6 \mathrm{DCA}-25$ particles. The materials are denoted " $\mathrm{nFe}^{0}-\mathrm{NH}_{2} \mathrm{PDMS}$-ratio" because only $n \mathrm{Fe}^{0}-6 \mathrm{DCA}-25$ were incorporated into the polymer. For example, the material with $n \mathrm{ne}^{0}-6 \mathrm{DCA}-25$ and a 0.7 carboxylic group to 1.0 amine group ratio is $\mathrm{nFe}^{0}-\mathrm{NH}_{2} \mathrm{PDMS}-70$.

\section{Materials characterization}

High resolution transmission electron microscopy (HRTEM) studies were carried out on a FEI Talos ${ }^{\mathrm{TM}}$ F200X TEM working at $80 \mathrm{kV}$. Samples were dropped from methanol suspensions onto $\mathrm{Cu}$ grids (No. 01843, Ted Pella, Inc.).

Scanning electron microscope (SEM) images were obtained on a UPRA ${ }^{\mathrm{TM}} 55 / 55 \mathrm{VP}$ microscope working at $20 \mathrm{kV}$ using the back-scattered detector mode. Samples for SEM measurements were prepared by depositing a drop of a dilute methanolic suspension of the material onto a magnetic carbon tape and allowing the drop to dry in the air.

The crystalline phases of the nanomaterials were assessed by X-ray diffraction (XRD) using an Ultima IV $\mathrm{X}$-ray diffractometer (Rigaku) equipped with a scintillator detector in Bragg-Brentano geometry working in the $3-90^{\circ}$ range and equipped with a high-power ceramic tube PW3373/10 LFF source with a $\mathrm{Cu}$ anode (1.54 $\AA$ ). The qualitative phase analysis was performed with the Jade software package supplied by Rigaku and compared to the ICDD-PDF-2 database.

The functional groups present in the nanoparticles were identified by their infrared (IR) signals using a PerkinElmer Fourier transform infrared (FTIR) spectrometer UATR-Two spectrophotometer equipped with a diamond crystal. 128 scans were averaged at $4 \mathrm{~cm}^{-1}$ resolution over $4000-400 \mathrm{~cm}^{-1}$.

Thermogravimetric analysis (TGA) measurements were performed on a TGA Q50 thermogravimetric analyser (TA Instruments, New Castle, DE) interfaced to a computer. Samples (3 mg) were heated at $10{ }^{\circ} \mathrm{C} \mathrm{min}{ }^{-1}$ under a flow at $10 \mathrm{~mL} \mathrm{~min}{ }^{-1}$ of $98 \%$ nitrogen $/ 2 \%$ oxygen from 50 to $600{ }^{\circ} \mathrm{C}$ with an initial equilibration time of $5 \mathrm{~min}$.

Raman measurements were made on a Renishaw Ramascope instrument equipped with a $10 \mathrm{~mW} 532 \mathrm{~nm}$ laser (SpectraPhysics) working at $1 \%$ power with a $30 \mathrm{~s}$ acquisition time, a Rayleigh filter, and a 1200 line $\mathrm{mm}^{-1}$ grating. The instrument was interfaced with an Olympus BH-2 microscope using a $10 \times$ objective. The detector was a SYNAPSE CCD model 354010 (HORIBA).

Rheological measurements were performed with an Anton Paar Physica MCR 301 strain-controlled rheometer equipped with a Peltier temperature-controller and parallel stainless steel plates ( $25 \mathrm{~mm}$ diameter) at $25 \pm 1{ }^{\circ} \mathrm{C}$. The data were collected using Rheoplus/32 Service V3.10 software. Before data were recorded, each sample was placed directly onto the base plate, and the upper plate was lowered to a $0.5 \mathrm{~mm}$ initial gap distance so that it was in contact with the upper sample surface. Then, any excess substrate sample was removed from the sides of the plates. Finally, the samples were left undisturbed at $25 \pm 1^{\circ} \mathrm{C}$ for $5 \mathrm{~min}$ to ensure thermal equilibration, and measurements began thereafter.

\section{Dehalogenation of HOCs by methanolic suspension of $n F e^{0}$}

All dehalogenation experiments (including those performed with nanoparticles embedded in polymers) were conducted in air on samples in closed glass vials containing $80 \mathrm{mM}$ (unless noted otherwise) of a halocarbon and $80 \mathrm{mM}$ of hexanes (as internal standard) in $5 \mathrm{~mL}$ of methanol. $100 \mathrm{mg}$ of $\mathrm{nFe}^{0}$ dry powder was added and the mixture was agitated throughout the experiment in a Branson 1210 ultrasonic bath at room temperature. Analyses during reaction periods were performed by placing a magnet next to the vial and then removing $1 \mu \mathrm{L}$ aliquot of the liquid that was injected directly into a gas chromatograph (GC) (vide infra). For reactions in which 
nanoparticles were reused, a new amount of halocarbon equivalent to the original amount, was added directly to the vial, and the reaction was continued.

Dehalogenation of HOCs by nanoparticles embedded in polymers

The concentrations of the halocarbons in the methanolic solutions were the same as above. However, $1 \mathrm{~g}$ of polymer containing $10 \%$ by weight of $\mathrm{nFe}^{0}$ was added instead of the $\mathrm{nFe}^{0}$ powder, and the mixture was not agitated. In addition, experiments using polymers without any nanoparticles were performed to follow the absorption of the HOCs into the polymer matrix.

\section{Determination of the HOC concentrations in solution}

A 5890A Hewlett Packard gas chromatograph equipped with a flame ionization detector and a ZB-624 Phenomenex ${ }^{\circledR}$ column $(20 \mathrm{~m} \times 0.25 \mathrm{~mm} ; 0.25 \mu \mathrm{m})$ at $200{ }^{\circ} \mathrm{C}$ (except for reactions with TCE, where the column temperature was $90{ }^{\circ} \mathrm{C}$ ) and with a $1.5 \mathrm{~mL} \mathrm{~min}^{-1}$ flow rate of $\mathrm{N}_{2}$ was used to analyze aliquots removed from the reaction mixtures. See Figure S1 for some typical chromatograms and Figure S2 for some calibration curves (see Supplementary Information (SI) for the Figures S1 and S2).

\section{Results and Discussion}

Particle characterization

All of the synthesized particles were magnetic. Figure S3 and 3 videos in the Supplementary Information show the magnetic responses of 3 types of particles as a powder or as a suspension in methanol. Some representative HRTEM micrographs of these $\mathrm{nFe}^{0}$ particles are shown in Figures 1 and S4 (SI section). Size distributions of individual particles could not be obtained as a result of their agglomeration on the $\mathrm{Cu}$ grids.

However, objects as small as 10,18 and $33 \mathrm{~nm}$ can be discerned for $\mathrm{nFe}^{0}-6 \mathrm{DCA}-60, \mathrm{nFe}^{0}-6 \mathrm{DCA}-25$, and $\mathrm{nFe}^{0}-6 \mathrm{DCA}-0$ samples, respectively. In addition, the core-shell structure of the particles is obvious. For example, in Figure 1, the shell thickness of the $\mathrm{nFe}^{0}-6 \mathrm{DCA}-25$ particles, ca. $4 \mathrm{~nm}$, is much larger than the length of a fully extended 6DCA molecule: the shell is multi-layered and/or it may contain a second less-dense component, such as an iron oxide. In addition, a change in the appearances of the particles was noted during their exposure to the electron beam; although the micrographs were recorded as rapidly as possible, some modification of the particle morphology

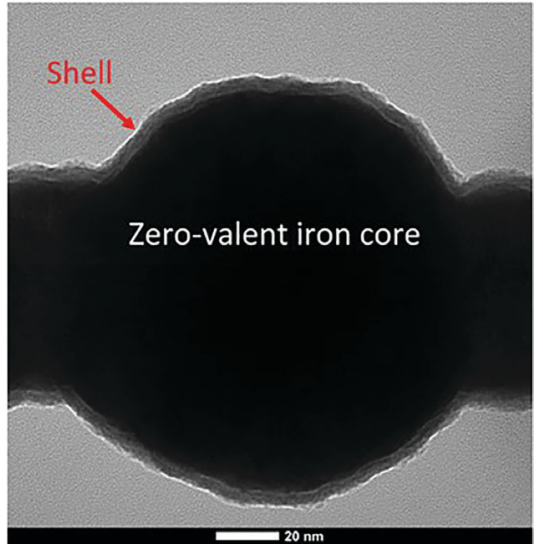

Figure 1. HRTEM micrograph of $\mathrm{nFe}^{0}-6 \mathrm{DCA}-25$.

may have occurred. Figures 2 and S5 (SI section) show the distribution of elements over a cross section of agglomerated particles. Notably, oxygen is detected clearly only in the shell of the individual $\mathrm{nFe} \mathrm{C}^{0}$-6DCA-25 species.

Figure 2 shows two STEM (scanning transmission electron microscope) micrographs for the $\mathrm{nFe}^{0}-6 \mathrm{DCA}-25$ particles and the spatial distribution of $\mathrm{Fe}$ and $\mathrm{O}$ atoms within them. The images were recorded with a high angle annular dark field (HAADF)-STEM detector which provides phase contrast for the particles but not the grid. ${ }^{31}$ Specifically not seen is evidence for the presence of $\mathrm{Cl}, \mathrm{S}, \mathrm{Cu}, \mathrm{Zn}$, and $\mathrm{Si}$; none of these was expected, and their absence gives some assurance that the micrographs present an accurate description of the $\mathrm{nFe}^{0}-6 \mathrm{DCA}-25$ particles. The presence of oxygen is consistent with the presence of iron oxide and/or the 6DCA coatings. However, due the intrinsic nature of this analytical technique, it was not possible to investigate the atomic distribution of the core of the particles, and the resolution does not permit a reasonable assessment of the size distributions. Regardless, objects as small as ca. $30 \mathrm{~nm}$ are clearly seen, and smaller ones are not measurable. Using X-ray diffractograms and Scherrer's method, the mean size of the iron cores for this sample is calculated to be ca. $4.5 \mathrm{~nm}$ (vide infra).

SEM images of the $\mathrm{nFe}^{0}$-6DCA particles synthesized at different temperatures are shown in Figure S6 (SI section). It is possible to discern that the particles have quasi-spherical shapes and that their sizes increase as the temperature at which they were prepared decreases. This trend is consistent with other measurements (vide infra).

XRD diffractograms of the same $n \mathrm{ne}^{0}$-6DCA particles are shown in Figure 3. Similar diffractograms were obtained for the $\mathrm{nFe}^{0}$ with different mDCAs (Figure S7, SI section). All exhibit three peaks, at $2 \theta=44.70^{\circ}, 65.02^{\circ}$ and $82.33^{\circ}$, that correspond to the (110), (200) and (211) planes of the body-centered cubic lattice reported for the $\alpha$-Fe phase. ${ }^{32}$ 

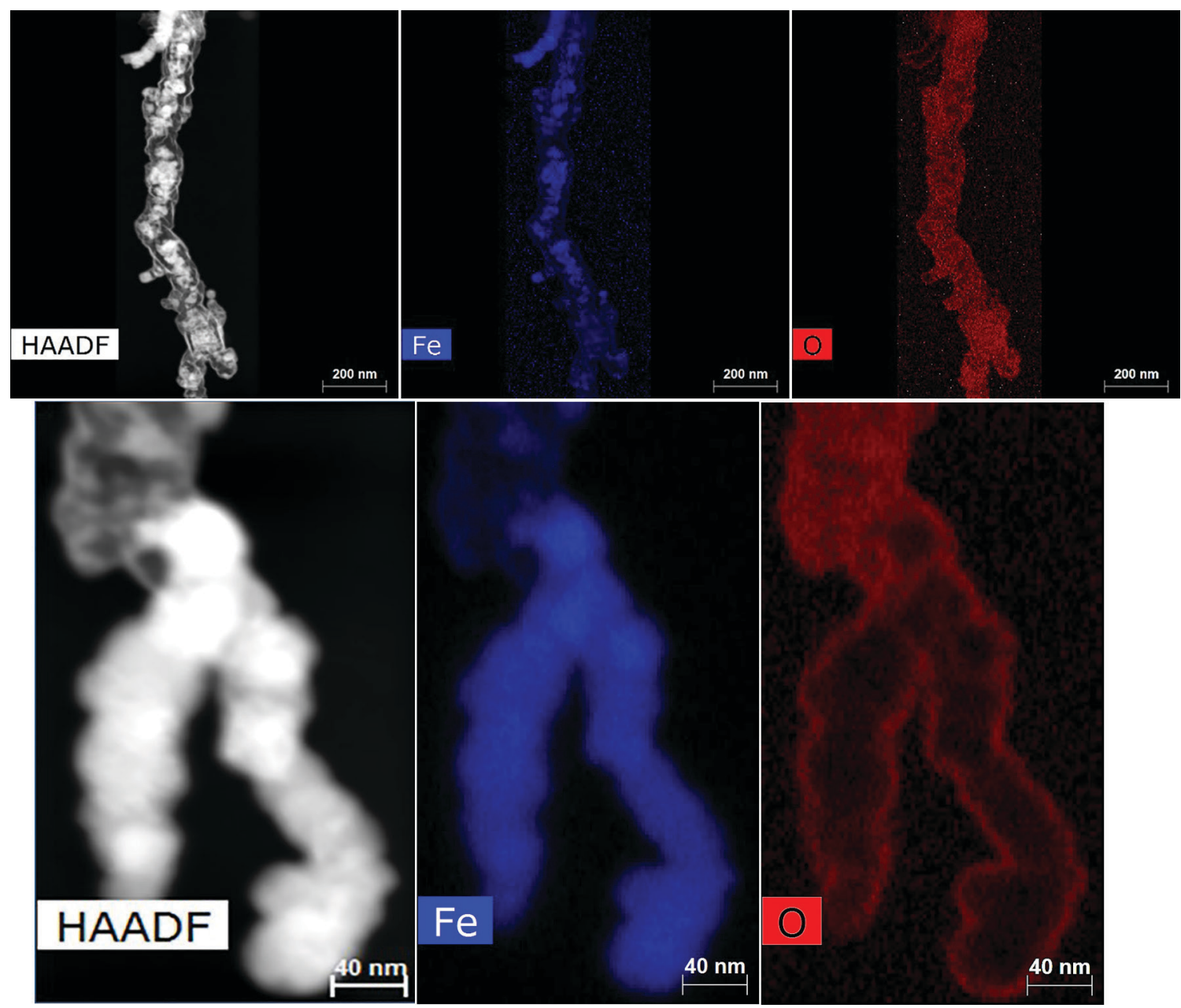

Figure 2. STEM micrographs of two different agglomerated $\mathrm{nFe}^{0}-6 \mathrm{DCA}-25$ particles on the sample grid (top and bottom), detected with a high angle annular dark field (HAADF) detector (left) and the spatial distributions of $\mathrm{Fe}$ (middle) and $\mathrm{O}$ (right).

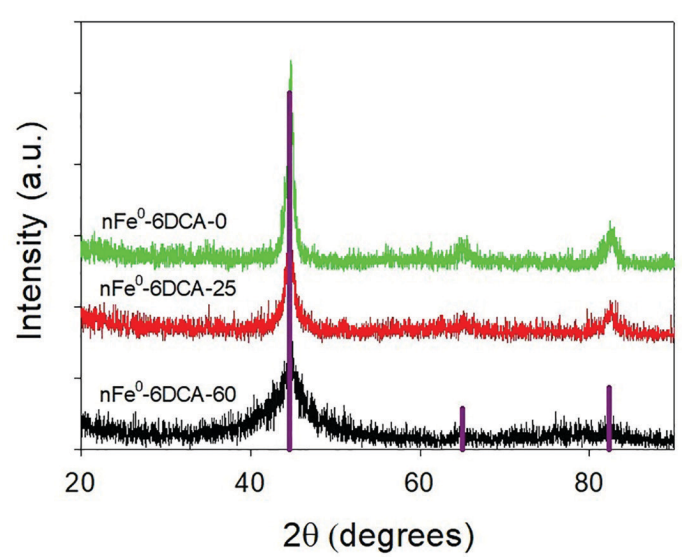

Figure 3. Vertically offset XRD diffractograms of the $\mathrm{nFe}^{0}-6 \mathrm{DCA}$ particles and of the $\alpha$-Fe phase (noted by vertical lines). ${ }^{32,33}$

The average crystallite sizes were estimated using the Scherrer's equation with $\lambda=0.154 \mathrm{~nm}$, assuming that the nanoparticles are completely spherical, a shape factor $\mathrm{k}=0.94$, and the full width at half maximum (FWHM) of the peaks at $2 \theta=44.70^{\circ} .{ }^{34}$
According to this analysis, particles made with different mDCAs at same temperature do not exhibit a linear relationship between their size and the length of the mDCA; all are near 4-5 $\mathrm{nm}$. However, the calculated sizes of the particles made by coating with 6DCA were temperature dependent: $6.1,4.5$ and $2.1 \mathrm{~nm}$ for $\mathrm{nFe}^{0}-0, \mathrm{nFe}^{0}-25$ and $\mathrm{nFe}^{0}-60$, respectively. As found in several other studies, ${ }^{35,36}$ smaller particles are formed at higher temperatures. This behavior may be due to larger increases in rates of nucleation than growth as temperature is increased.

Raman spectra of the materials coated with 6DCA at different temperatures are shown in Figure S8 (SI section). As expected, ${ }^{37}$ the $\mathrm{nFe}{ }^{0}-0$ sample does not have peaks in the $200-800 \mathrm{~cm}^{-1}$ region. Although the other two samples have at least two broad peaks between 300 and $500 \mathrm{~cm}^{-1}$, which may be from an iron oxide phase or (oxy)hydroxides; ${ }^{38}$ it was not possible to assign them. The Raman results suggest that formation of iron oxide phases is promoted by higher temperatures. It is known that $\mathrm{nFe}^{0}$ particles are sensitive to air oxidation when heated, and the iron oxides are 
susceptible to further oxidation under some conditions. ${ }^{38,39}$ Indeed, TGA analyses show that these materials are oxidized upon heating (vide infra). Thus, the new peaks may be due to in situ oxidation of the $\mathrm{nFe}^{0}$ particles even during the Raman analyses.

The attenuated total reflectance (ATR) FTIR method employed to record the spectra in Figure 4 is most sensitive to functional groups very near the particle surfaces. The peaks at 2896 and $2832 \mathrm{~cm}^{-1}$ correspond to stretching motions of the $-\mathrm{CH}_{2}-$ groups $^{40}$ and those at 1000 and $808 \mathrm{~cm}^{-1}$ are from the $-\mathrm{COOH}$ of 6DCA adsorbed on the surface of the materials. The lack of significant absorptions near $3400 \mathrm{~cm}^{-1}$ and ca. 654 and $559 \mathrm{~cm}^{-1}$ suggests (but does not prove) the absence of surface $\mathrm{O}-\mathrm{H}$ groups that could arise from atmospheric oxidation of iron atoms. The FTIR spectrum for $\mathrm{nFe}^{0}$-12DCA (Figure $\mathrm{S} 9$, SI section) is a similar to the ones presented above.

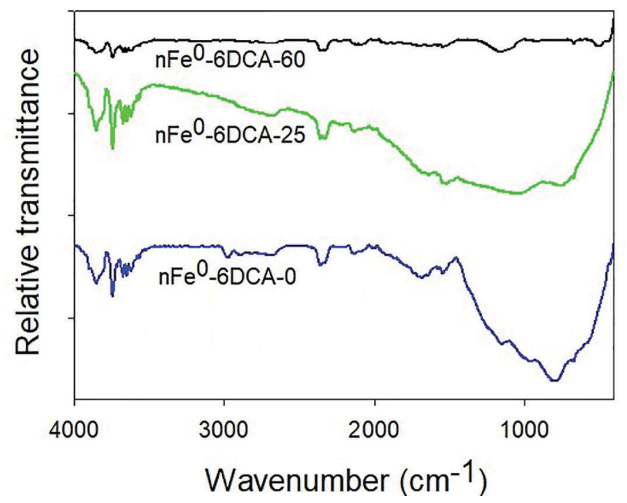

Figure 4. Vertically offset FTIR spectra of the $\mathrm{nFe}^{0}$-6DCA particles made at different temperatures.

The thermograms in Figure S10 (SI section) from TGA analyses of the three 6DCA samples show characteristic behavior of $\mathrm{nFe}^{0}$ nanoparticles. ${ }^{25,33}$ They gain weight as a result of oxidation as they are heated under a $98 \% \mathrm{~N}_{2}-2 \%$ $\mathrm{O}_{2}$ atmosphere; the expected weight loss from pyrolysis of the organic matter adsorbed on the nanoparticle surfaces appears to be masked by the weight gain upon oxidation that may be occurring between 50 and $200^{\circ} \mathrm{C}$, and at still higher temperatures. The first derivatives of the corresponding TGA curves, shown at the top of Figure S10 (SI section), provide evidence for possible transitions at ca. $230{ }^{\circ} \mathrm{C}$ and ca. $465{ }^{\circ} \mathrm{C}$ which may be related to the oxidation of iron atoms at interior sites. Indeed, the first-derivative peak at ca. $465^{\circ} \mathrm{C}$ is consistent with the known $\gamma-\mathrm{Fe}_{2} \mathrm{O}_{3} \rightarrow \alpha-\mathrm{Fe}_{2} \mathrm{O}_{3}$ transition. ${ }^{39}$

The TGA of $\mathrm{nFe}^{0}$-12DCA (Figure S11, SI section) is very similar to those in Figure S10 (SI section). Furthermore, XRD diffractograms and ATR FTIR spectra of the nanoparticles made with 6DCA indicate the formation of hematite $\left(\alpha-\mathrm{Fe}_{2} \mathrm{O}_{3}\right)^{41}$ after heating (Figures S12 and S13, SI section).

Using Scherrer's equation and the XRD peak at $33.2^{\circ}$ for the hematite phase, the average sizes of the $\mathrm{Fe}^{0}-0$, $\mathrm{nFe}^{0}-25$ and $\mathrm{nFe}^{0}-60$ particles that had been heated to $600{ }^{\circ} \mathrm{C}$ are 17.4, 34.1 and $23.4 \mathrm{~nm}$, respectively. Increases in the crystallite size upon heating have been observed for several types of nanoparticles, and they have been attributed to calcination of iron oxide nanoparticles or nanocomposites. ${ }^{42}$ Note also that although peaks at ca. 650 and $550 \mathrm{~cm}^{-1}$ (corresponding to the $\mathrm{Fe}-\mathrm{O}$ bond) and at ca. $3250 \mathrm{~cm}^{-1}$ (from -OH stretching) are present in the ATR FTIR spectra, peaks at ca. 2890 and $2830 \mathrm{~cm}^{-1}$ (corresponding to the $-\mathrm{CH}_{2}$ modes from the initial $\mathrm{nFe}^{0}$ ) are absent; presumably, the 6DCA originally on the surfaces of the nanoparticles has been pyrolyzed. The differences may be due to the effect of excluding the contribution from loss of the acid, which would increase the ratio. Using this difference, the maximum weight percentage of 6DCA contributing to the particle weight of each particle type was estimated to be 2,6 and $14 \%$ for $\mathrm{nFe}^{0}-0, \mathrm{nFe}^{0}-25$ and $\mathrm{nFe}^{0}-60$, respectively.

Thus, the sizes can be controlled by the temperature at which the particles are formed, and the 6DCA (as well as the others mDCAs) adsorbed on the surfaces does protect the particles from air oxidation at room temperature, but not at elevated temperatures.

Degradation of trichloroethylene (TCE) in methanol suspensions of $\mathrm{nFe}^{0}$ particles

All the particles are stable in methanolic suspension for more than $48 \mathrm{~h}$ except $\mathrm{nFe}^{0}$-2DCA which slowly dissolved in the solvent. The dechlorination of TCE by different $n \mathrm{Fe}^{0}$ particles has been demonstrated by others in aqueous media. ${ }^{43,44}$ Methanol is the solvent employed here for reasons of ease of analysis. Although it was possible to follow the loss of TCE (Figure 5), no products could be detected by our GC; at least five chlorinated intermediate by-products have been identified in other studies. ${ }^{44}$

All of the $\mathrm{nFe}^{0}$-mDCA particle types were able to degrade TCE. However, they were able to do so with significantly different efficiencies that do not correlate with the length of the mDCA chains. $\mathrm{nFe}^{0}-6 \mathrm{DCA}$ provided the highest degradation yield, reaching ca. $76 \%$ TCE loss over a period of $10 \mathrm{~h}$ under the conditions employed. In order to obtain more quantitative data and to facilitate the analyses, our experiments have focused on dehalogenation of molecules whose degradation products are easier to follow by our GC analyses using different formulations of $\mathrm{nFe}^{0}-6 \mathrm{DCA}$ as the nanoparticles. 


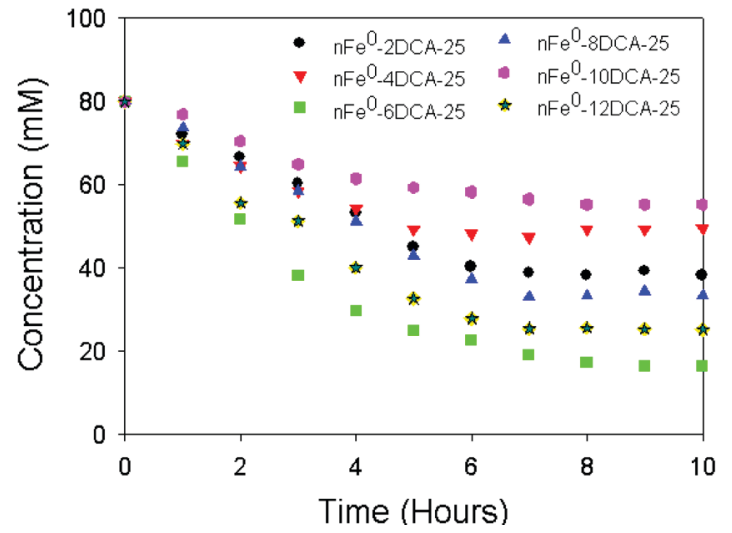

Figure 5. Loss of TCE (initially $80 \mathrm{mM}$ ) in $5 \mathrm{~mL}$ methanol as a function of time in the presence of $100 \mathrm{mg}$ of $n \mathrm{Fe}^{0}$-mDCA- 25 powders with $\mathrm{m}=2$, $4,6,8,10$, and 12 .

Unfortunately, experiments to dechlorinate trichloroacetic acid, dichloroacetic acid, and chloroacetic acid using the $\mathrm{nFe}^{0}$ particles were unsuccessful. The particles dissolved in each of them due to replacement of the mDCAs on the surfaces by the more acidic chlorinated acids: the $\mathrm{p} K_{\mathrm{a}}$ values for the tri-, di-, and mono-chlorinated acids are $0.66,1.35$ and 2.82 , respectively; those of the mDCAs are in the 4-5 range.

Dehalogenation of HOCs in methanol by suspensions of $n \mathrm{ne}^{0}-6 \mathrm{DCA}$ particles

Dehalogenations of bromobenzene and chlorobenzene by the particles were examined in detail. Benzene was the sole product detected in both cases. The results using $\mathrm{nFe} \mathrm{f}^{0}$-6DCA-25 are shown in Figure 6. For both substrates, complete dehalogenation occurred within $16 \mathrm{~h}$ under the experimental conditions employed. As noted for several other substrates the rates the dehalogenation by $\mathrm{nFe}^{0},{ }^{45,46}$ can be expressed in terms of pseudo first-order reactions. The apparent rate constants $(\mathrm{k})$ are $0.34 \pm 0.04$ and $0.17 \pm 0.01 \mathrm{~h}^{-1}$ for the brominated and chlorinated aromatic molecules, respectively. Although they have only comparative significance, they do demonstrate that, as expected, debromination is faster than dechlorination. Also, debromination is faster than dechlorination when bromobenzene and chlorobenzene are reacted in one solution (Figure S17, SI section).

Analogous results, but at a faster rate, were obtained in the deiodination of iodobenzene (Figure S14, SI section). Methyl bromoacetate and ethyl iodoacetate were also dehalogenated by the nanoparticles (Figure S15, SI section). However, the $\mathrm{nFe}^{0}$ materials were not able to convert fluorobenzene to benzene under the same reaction conditions; a $4 \%$ decrease of fluorobenzene was observed without any detectable benzene in the methanol
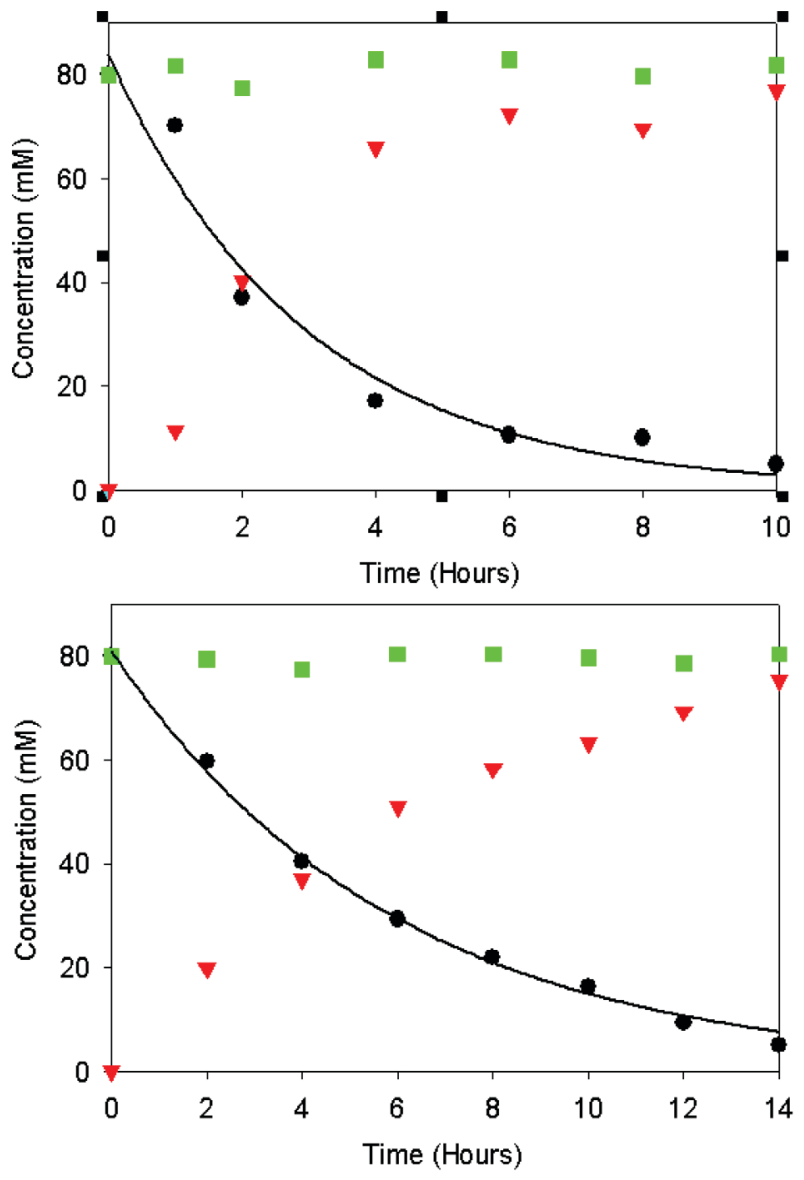

Figure 6. Decreases in initial $80 \mathrm{mM}$ concentrations of bromobenzene (top) or chlorobenzene (bottom) (as circles) in $5 \mathrm{~mL}$ of methanol and formation of benzene (as triangles) as a function of time in the presence of $100 \mathrm{mg} \mathrm{nFe} \mathrm{e}^{0}-6 \mathrm{DCA}-25$. The curves are the best fits of the halocarbon loss data to a single exponential function. The sums of the halobenzene and benzene concentrations are shown as squares.

(data not shown). Consistent with this observation, 1-chloro-3-fluorobenzene was dechlorinated, without any detectable loss of fluorine (Figure S16, SI section), and neither 2-fluoro-4-methylpyridine nor 4-fluorobenzonitrile underwent any apparent reaction (data not shown).

In another experiment, intended to examine the effectiveness of the $\mathrm{nFe}^{0}$-6DCA-25 nanoparticles when subsequent aliquots of a haloaromatic are added to a reaction mixture, it was found that the rate of dehalogenation of bromobenzene was constant throughout 3 runs, but that for chlorobenzene appeared to slow a small amount (Figure S18, SI section); the second and third runs had $\mathrm{k}=0.14 \pm 0.01$ and $0.12 \pm 0.01 \mathrm{~h}^{-1}$, respectively.

The relative rates of dehalogenation were also determined with equal weights of the 3 types of $n \mathrm{Fe}^{0}$-6DCA nanoparticles (Figure S19, SI section). Although debromination occurred at the same rate, regardless of the nanoparticle type, the relative rates for dechlorination were $0.29 \pm 0.01,0.17 \pm 0.01$, and $0.13 \pm 0.01 \mathrm{~h}^{-1}$ for $\mathrm{nFe}^{0}-6 \mathrm{DCA}-60, \mathrm{nFe}^{0}-6 \mathrm{DCA}-25$, and 
$n \mathrm{ne}^{0}-6 \mathrm{DCA}-0$, respectively. Tentatively, we ascribe the lack of effect on the debromination to its lower activation energy. It may be diffusion controlled, whereas dechlorination depends on the surface area of the nanoparticles as well as diffusion. For that reason, fewer collisions of a nanoparticle with a chlorobenzene molecule than with a bromobenzene molecule are effective in leading to loss of a halogen atom.

According to the accepted mechanism for dehalogenation by $\mathrm{nFe}^{0}$, oxidation of one atom of iron is needed for removal of one atom of halogen. ${ }^{47}$ The experiments above were performed with a larger total number of iron atoms in the nanoparticles than the number to chlorine or bromide atoms in the substrates. Results from experiments with a 1:1 atomic ratio for degradation of chlorobenzene are shown in Figure 7; data for the analogous experiments with bromobenzene are in Figure S20 (SI section). The $\mathrm{k}$ values for $\mathrm{nFe}^{0}-6 \mathrm{DCA}-0, \mathrm{nFe}^{0}-6 \mathrm{DCA}-25$ and $\mathrm{nFe}{ }^{0}-6 \mathrm{DCA}-60$ are $0.08 \pm 0.01,0.095 \pm 0.12$ and $0.032 \pm 0.005 \mathrm{~h}^{-1}$, respectively, do not show a clear trend. However, the corresponding yields of dechlorination, 40, 53 and $74 \%$ indicate that the smaller the $\mathrm{nFe}^{0}$ particle, the more effective it is at removing a chlorine atom. By contrast, debromination proceeded to ca. $83 \%$ with $\mathrm{k}=0.063 \pm 0.007 \mathrm{~h}^{-1}$ for the three nanoparticles.

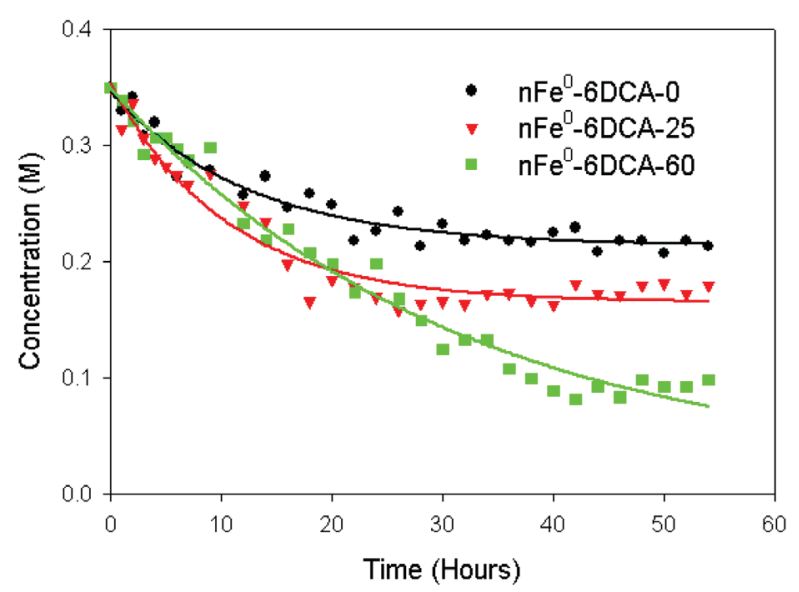

Figure 7. Dechlorination of $0.35 \mathrm{M}$ chlorobenzene in $5 \mathrm{~mL}$ of methanol as a function of time using $100 \mathrm{mg}$ of $\mathrm{nFe}^{0}$-6DCA particles synthesized at different temperatures. The curved lines are the best fits of chlorobenzene loss to a single exponential function.

XRD analyses of the powders recovered after dechlorination of chlorobenzene at $1: 1 \mathrm{Fe}: \mathrm{Cl}$ atom ratios using $\mathrm{nFe}^{0}-6 \mathrm{DCA}-60$ demonstrate the formation of a different crystalline lattice from the initial one: goethite $(\gamma-\mathrm{FeO}(\mathrm{OH}))$, but probably with another iron oxy-hydroxide phase (Figure S21, SI section). ${ }^{48}$ Using Scherrer's method, the sizes of the $\mathrm{nFe}^{0}$ particles obtained with 6DCA as the stabilizer at different temperatures, after the reaction, are calculated to be $\geq 11 \mathrm{~nm}$. Goethite has been reported to be one of the final $\mathrm{nFe}^{0}$ phases from reduction of $\mathrm{Cr}^{\mathrm{VI}}$ and $\mathrm{Pb}^{\mathrm{II}}{ }^{49}$ Because goethite can be transformed into a zero-valent iron by heating it in a hydrogen flow, ${ }^{50}$ it is possible, at least in theory, to regenerate the zero-valent iron phase for reduction of additional halocarbons.

\section{$n F e^{0}-6 D C A-25$ particles embedded in $\mathrm{NH}_{2} \mathrm{PDMS}-4 \mathrm{DCA}$}

The concentration of monomers with 3-aminoproyl groups in $\mathrm{NH}_{2} \mathrm{PDMS}$ is calculated to be ca. 0.9 mmoles per gram (see Supplementary Information). Thus, materials consisting of $\mathrm{NH}_{2} \mathrm{PDMS}$ with $4 \mathrm{DCA}$ in $\mathrm{COOH} / \mathrm{NH}_{2}$ group ratios of $100,70,50$ and $20 \%$ were prepared. There is a marked change in the viscosity of the polymers after addition of one equivalent of 4DCA and then upon further addition of 10\% of nanoparticles (Figure S22, SI section). Because of the inclusion of the $\mathrm{nFe}^{0}$ particles, these polymers respond when placed in the proximity of a magnet; see video 1 in the Supplementary Information for a demonstration of this behavior.

As reported by He et al. ${ }^{28}$ IR spectra showed no evidence for the presence of free $-\mathrm{NH}_{2}\left(\mathrm{ca} .1570 \mathrm{~cm}^{-1}\right)$ in the IR spectra of these samples (Figure 8); other characteristic signals at ca. $1020 \mathrm{~cm}^{-1}$ for $\mathrm{Si}-\mathrm{O}-\mathrm{Si}$ stretching and at ca. $805 \mathrm{~cm}^{-1}$ attributed to $-\mathrm{CH}_{3}$ and $\mathrm{Si}-\mathrm{C}$ stretching are apparent. $^{51}$

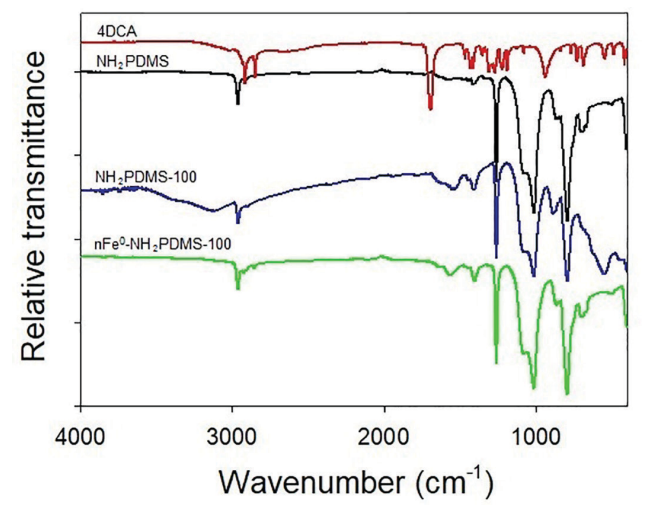

Figure 8. Vertically offset IR spectra of 4DCA (red), $\mathrm{NH}_{2} \mathrm{PDMS}$ (black), $\mathrm{NH}_{2} \mathrm{PDMS}-100$ (blue) and $\mathrm{nFe}^{0}-\mathrm{NH}_{2} \mathrm{PDMS}-100$ (green).

Also, the absence in the IR spectrum of $\mathrm{NH}_{2} \mathrm{PDMS}-100$ of a strong peak at ca. $1704 \mathrm{~cm}^{-1}$ from the $\mathrm{C}=\mathrm{O}$ stretching mode of 4DCA, with and without nanoparticles, indicates that the vast majority of the acid has reacted to create ammonium carboxylate inter-chain (as well as some intra-chain) crosslinks. The acid peak is not observable in the spectra of other compositions in which the $\mathrm{COOH} / \mathrm{NH}_{2}$ ratios are $<100 \%$ (results not shown). However, a new broad peak at ca. $1562 \mathrm{~cm}^{-1}$, which is attributed to $-\mathrm{NH}_{3}{ }^{+}, 52$ is present. 
The $n F^{0}-6 D C A-25$ nanoparticles from $n \mathrm{ne}^{0}-\mathrm{NH}_{2} \mathrm{PDMS}-100$ were analyzed by Raman and XRD (Figure S23, SI section) after being recovered by dissolving the polymer in an acetone:pentane mixture. The data demonstrate that the Fe phase does not change when the nanoparticles are incorporated into the polymer.

Quantification of the viscoelastic changes upon addition of 4DCA to the $\mathrm{NH}_{2}$ PDMS were measured by rheology. The steady-shear viscosities with acid are much larger than that of $\mathrm{NH}_{2} \mathrm{PDMS}$ alone (Figure S24, SI section). Also, as expected, the increase in viscosity is not linear with respect to the increase in the $\mathrm{COOH} / \mathrm{NH}_{2}$ group ratio. Somewhat surprisingly,,$^{53}$ the viscosity actually was reduced by addition of nanoparticles to an acid-crosslinked polymer sample. However, similar behavior in different polymer matrices (as well as ones in which the viscosity increases! $)^{54,55}$ has been reported..$^{56,57}$

The storage (G') and loss (G') moduli from frequency and strain sweeps for all of the $\mathrm{NH}_{2} \mathrm{PDMS}$ samples with different amounts of 4DCA are collected in Figures S24 and S25 (SI section). The data show that both samples in which the $\mathrm{COOH} / \mathrm{NH}_{2}$ ratio is $100 \%$ are not gels: in the linear viscoelastic regions, G' remains lower than G'. However, the materials do become gel-like when smaller amounts of 4DCA are added.

Upon addition of $10 \mathrm{wt} . \%$ of $\mathrm{nFe}^{0}-6 \mathrm{DCA}-25$ particles to the polymers at a $\mathrm{COOH} / \mathrm{NH}_{2}$ ratio of $70 \%$, G' exceeds $G$ " at lower frequencies only (Figure 9). Similar behavior is observed at $\mathrm{COOH} / \mathrm{NH}_{2}$ ratios of $20 \%$. Because the crossing point between G' and G" for both of these samples is near the same angular frequency, their relaxation times are similar. However, the matrix with $\mathrm{nFe}^{0}-6 \mathrm{DCA}-25$ particles and a $\mathrm{COOH} / \mathrm{NH}_{2}$ ratio of $50 \%$ remained gel-like throughout the frequency region explored: G' remains higher than G". Also, the addition of the nanoparticles to an $\mathrm{NH}_{2}$ PDMS-4DCA with different ratios does not appear to promote gelation and it decreases the viscosity (Figure S25, SI section).

\section{Dehalogenation of bromobenzene and chlorobenzene by} $\mathrm{nFe} \mathrm{e}^{0}-6 \mathrm{DCA}-25$ embedded in polymeric matrices

Dehalogenation of bromobenzene and chlorobenzene in methanolic solutions in the presence of polymers containing $\mathrm{nFe}^{0}-6 \mathrm{DCA}$ nanoparticles proceeded quite efficiently. Although for reasons of polarity, methanol does not enter the polymer to a significant extent (and water does to an even lesser extent), ${ }^{27}$ the halobenzenes are able to diffuse readily into and from it. Results from experiments performed with $\mathrm{NH}_{2} \mathrm{PDMS}-50$, including evidence for the ability of the halobenzenes to enter the polymer matrices,
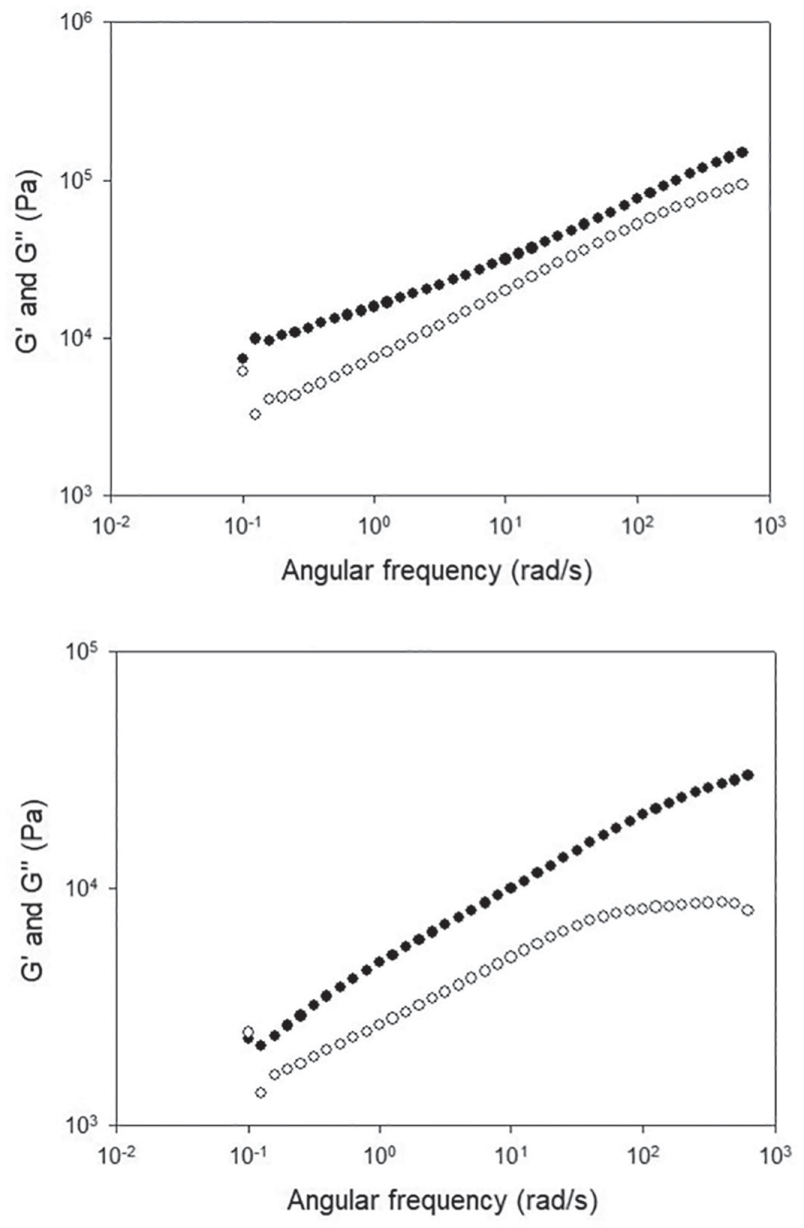

Figure 9. Storage (G'; $)$ and loss $\left(\mathrm{G}^{\prime \prime} ; \bigcirc\right)$ moduli at $1 \%$ strain of $\mathrm{NH}_{2}$ PDMS-50 without (top) and with (bottom) $10 \mathrm{wt} . \% \mathrm{nFe}^{0}$-6DCA-25.

are shown in Figure 10; more extensive sets of results are collected in Figures S27 and S28 (SI section) for chlorobenzene and bromobenzene, respectively.

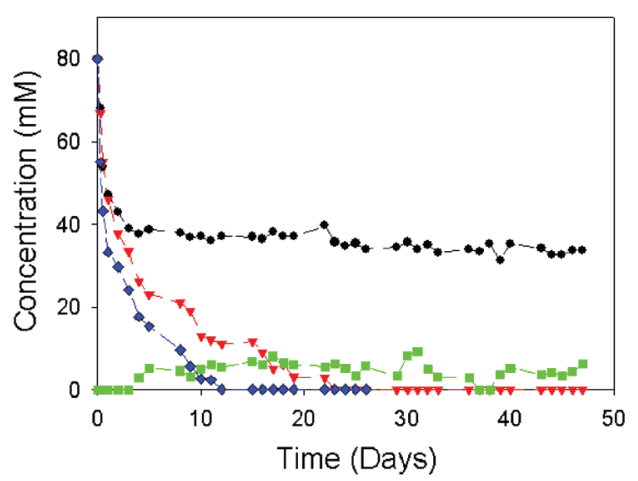

Figure 10. Temporal changes in concentrations detectable in the liquid portions of initially $80 \mathrm{mM}$ chlorobenzene (circles) and $80 \mathrm{mM}$ benzene (diamonds) in $5 \mathrm{~mL}$ of methanol containing $1 \mathrm{~g}$ of $\mathrm{NH}_{2}$ PDMS-50 and for $80 \mathrm{mM}$ chlorobenzene (triangles) and initially $0 \mathrm{mM}$ benzene (squares) in $5 \mathrm{~mL}$ of methanol containing $1 \mathrm{~g}$ of $\mathrm{nFe}_{0} \mathrm{NH}_{2} \mathrm{PDMS}-50$.

The first step in this dehalogenation process is the absorption of a part of the halobenzene into the 
polymeric matrix, so that it can come into contact with the $\mathrm{nFe}^{0}$ particles. As shown in Figure 10, ca. 50\% of the chlorobenzene originally in the methanol was transferred to the polymer. Results from a similar experiment show that almost all of the benzene produced after dehalogenation was absorbed by the polymer.

As shown by the red triangles in Figure 10, the sorbed chlorobenzene within the polymer containing the $\mathrm{nFe}^{0}$ particles is converted to benzene over time. Because the dehalogenation analyses were performed on aliquots taken from the methanol layer, it is not possible to relate directly the percent conversion of the chlorobenzene to the concentrations indicated; a part may be unreacted but held within the polymer. However, the fact that no chlorobenzene can be detected in the methanol at long times, combined with the solubilities determined in the two phases in the absence of the nanoparticles, demonstrates that reaction was virtually complete at long times; the detection limit of our GC analysis is $2 \mathrm{mM}$. As it can be seen in Figure S28 (SI section), the absorption and dehalogenation profiles of bromobenzene under the same conditions are similar, although more rapid, than those of chlorobenzene. In fact, the simultaneous dehalogenation of $40 \mathrm{mM}$ of each, bromobenzene and chlorobenzene in methanol under the same conditions (mentioned above), again indicate the more rapid dehalogenation of bromobenzene than of chlorobenzene (Figure S29, SI section).

Results from the dehalogenation of $80 \mathrm{mM}$ TCE under the same experimental conditions in the presence of $\mathrm{NH}_{2} \mathrm{PDMS}-50$ are shown in Figure S30 (SI section). The initial reduction in the TCE concentration within the methanol liquid is attributed again to its absorption into the polymer. Over longer times, it is followed by dechlorination by $\mathrm{nFe}^{0}$ particles in the polymer. Due to the very volatile nature of all of the expected dechlorinated products, we were unable to detect them using our GC analyses either in this experiment or (as mentioned above) when the $\mathrm{nFe}^{0}$ particles were suspended in the methanol alone.

To determine if any unreacted halobenzene remained at long times inside the polymers containing $\mathrm{nFe}^{0}$ particles, the polymer was removed from the original methanol and resuspended with stirring in pure methanol for $48 \mathrm{~h}$ at room temperature. No bromobenzene or chlorobenzene was detectable in the new aliquots, although benzene was detected in both cases.

Figure 11 shows the changes in appearance of $n \mathrm{ne}^{0}-\mathrm{NH}_{2} \mathrm{PDMS}-50$ at various times during the dechlorination of chlorobenzene. The color change from black to brown is indicative of the transition from the $\alpha-\mathrm{Fe}$ of the $\mathrm{nFe}^{0}$ to goethite of the particles inside the polymer (vide supra).

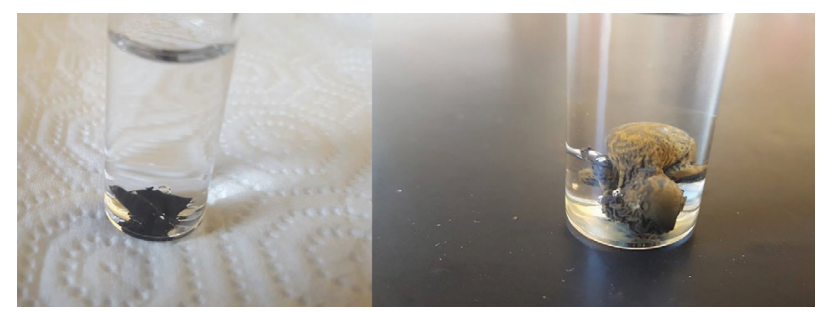

Figure 11. Appearances of a $\mathrm{nFe}^{0}-\mathrm{NH}_{2} \mathrm{PDMS}-50$ polymer during the dechlorination of chlorobenzene under the conditions described in Figure 10 after 0 (left) and 25 days (right) of reaction.

Mechanical properties of $\mathrm{NH}_{2} \mathrm{PDMS}$ crosslinked by 10 wt.\% $n F e^{0}-6 D C A-25$ particles (in the absence of 4DCA)

Although no dehalogenation experiments were conducted with a material prepared by embedding 10 wt. $\% \mathrm{nFe}^{0}$-6DCA-25 particles into an $\mathrm{NH}_{2}$ PDMS matrix, its elastic properties are worthy of mention because this material and others like it may be useful for future applications. A $0.5 \mathrm{~mm}$ thick film on the $2.5 \mathrm{~cm}$ diameter parallel plates of our rheometer could be extended to $10 \mathrm{~cm}$ in length and reduced to ca. $0.4 \mathrm{~cm}$ diameter (video 4), and then recompressed. The quantitative changes in the force exerted on the instrument by the expansion/contraction, up to (and beyond) the instrumental measurement limit of 50 N, are displayed in Figure S31 (SI section).

\section{Conclusions}

We have developed a wet-synthesis method to obtain air-stable, zero-valent iron nanoparticles that are coated with alkanedioic acids. We demonstrate that they can be employed alone or incorporated into appropriate polymeric matrices, the case explored being an aminopolydimethylsiloxane, to dehalogenate a variety of chlorinated, brominated and iodinated molecules. Although the temperature at which the particles are produced upon reduction of the precursor $\mathrm{Fe}^{2+}$ ions is an important parameter in determining their size, all consist almost exclusively of $\mathrm{Fe}^{0}$ with a coating of an mDCA, especially 6DCA, and all are protected from oxidation by the air. However, upon being heated, the iron in the nanoparticles changes its phase to hematite.

Embedding the nanoparticles into the polymer matrices was accomplished without altering the $\mathrm{nFe}^{0}$ phase and their dehalogenating ability remains intact. Due to its high solubility within the polymer, a large amount of the benzene product from dehalogenation of the halobenzenes is retained within the polysiloxane matrices. Interestingly, introduction of the particles in the polymer reduced the viscosity of the matrices without their becoming gel-like. 
Future studies will be performed in aqueous media (where the nanoparticles are also stable), including in bodies of water where HOCs are known to be pollutants. In fact, we expect that the materials developed in this study will be able to remediate pollution in various types of environments. The low cost of their production and their high stability in air and water make these materials attractive for pollution remediation in the real world. In fact, the procedures outlined here are easily amenable to the production of a variety of related materials by changing the dicarboxylic acids employed to coat the $\mathrm{Fe}^{0}$ for crosslinking and by using other polysiloxanes (or completely different polymers) with different molecular weights or degrees of amino substitution.

\section{Supplementary Information}

Supplementary Information (SI) available: chromatograms, calibration curves, photographs showing the magnetic response of the particles, TEM and SEM images of the particles, Raman spectra, XRD patterns and IR spectra of the obtained materials before and after heating, figures showing the dechlorination of 1-chloro-3-fluorobenzene, simultaneous dehalogenation of bromobenzene and chlorobenzene, re-use of the $\mathrm{nFe}^{0}$ particles in suspensions for dehalogenations, effects of size of the nanoparticles on the degradation of halobenzenes, $\mathrm{X}$-ray diffractograms of the nanoparticles after the dehalogenation process, typical appearances of the gel-like materials, viscosities and G' and G" values of different polymer matrices, results from absorption/degradation of chlorobenzene and bromobenzene using particles embedded in a polymer matrix, pictures of changes in the appearance of particles before, during and after reaction, calculation of the number of moles of amino groups per mole of monomer units in $\mathrm{NH}_{2} \mathrm{PDMS}, 3$ videos of the magnetic responses of 3 different types of the materials, and a video of the extension of a polysiloxane containing only particles as cross-linkers. All these data are available free of charge at http://jbcs.sbq.org.br.

\section{Acknowledgments}

The authors thank the Georgetown Environment Initiative for its support of this research and the National Science Foundation MRI Program for acquisition of the Raman instrument used in these studies (NSF CHE-1429079). Prof Vijay John from Tulane University is thanked for useful discussions. At Georgetown, we thank Dr Faye Rubinson for providing the magnetic tape used to obtain the SEM images and Prof Kaveh Jorabchi for supplying some of the halocarbons examined. Dr Christina Brantner of the George Washington University Nanofabrication and Imaging Center is acknowledged for help recording the TEM images.

\section{References}

1. Liu, X.; Chen, J.; Zhang, J.; Ind. Eng. Chem. Res. 2008, 47, 5362.

2. Dai, Y.; Song, Y.; Wang, S.; Yuan, Y.; Water Res. 2015, 71, 64.

3. Navarro, I.; de la Torre, A.; Sanz, P.; Pro, J.; Carbonell, G.; Martínez, M. Á.; Environ. Res. 2016, 149, 32.

4. Weijs, L.; Briels, N.; Adams, D. H.; Lepoint, G.; Das, K.; Blust, R.; Covaci, A.; Environ. Res. 2015, 137, 199.

5. Barón, E.; Giménez, J.; Verborgh, P.; Gauffier, P.; de Stephanis, R.; Eljarrat, E.; Barceló, D.; Environ. Pollut. 2015, 203, 107.

6. Pourmoghaddas, H.; Stevens, A. A.; Water Res. 1995, 29, 2059.

7. Adin, A.; Katzhendler, J.; Alkaslassy, D.; Rav-Acha, C.; Water Res. 1991, 25, 797.

8. Wilkins, J. R.; Reiches, N. A.; Kruse, C. W.; Am. J. Epidemiol. 1979, 110, 420.

9. Rexwinkel, G.; Heesink, B. B. M.; Van Swaaij, W. P. M.; J. Chem. Eng. Data 1999, 44, 1139.

10. Schnoor, J. L.; Environ. Sci. Technol. 2010, 44, 2767.

11. Balcke, G. U.; Turunen, L. P.; Geyer, R.; Wenderoth, D. F.; Schlosser, D.; FEMS Microbiol. Ecol. 2004, 49, 109.

12. Ghaffar, A.; Tabata, M.; Appl. Catal., B 2009, 86, 152.

13. Ghaffar, A.; Tabata, M.; Waste Manage. 2009, 29, 3004.

14. Chekli, L.; Bayatsarmadi, B.; Sekine, R.; Sarkar, B.; Shen, A. M.; Scheckel, K. G.; Skinner, W.; Naidu, R.; Shon, H. K.; Lombi, E.; Donner, E.; Anal. Chim. Acta 2016, 903, 13.

15. Zhang, W. X.; US Patent No. 7128841 B2 2006.

16. Bezbaruah, A. N.; Chisholm, B. J.; Krajangpan, S.; US Patent No. 004325 A1 2001.

17. Zhao, X.; Liu, W.; Cai, Z.; Han, B.; Qian, T.; Zhao, D.; Water Res. 2016, 100, 245.

18. Wang, C. B.; Zhang, W. X.; Environ. Sci. Technol. 1997, 31, 2154.

19. Ponder, S. M.; Darab, J. G.; Bucher, J.; Caulder, D.; Craig, I.; Davis, L.; Edelstein, N.; Lukens, W.; Nitsche, H.; Rao, L.; Shuh, D. K.; Mallouk, T. E.; Chem. Mater. 2001, 13, 479.

20. Nurmi, J. T.; Tratnyek, P. G.; Sarathy, V.; Baer, D. R.; Amonette, J. E.; Pecher, K.; Wang, C.; Linehan, J. C.; Matson, D. W.; Penn, R. L.; Driessen, M. D.; Environ. Sci. Technol. 2005, 39, 1221.

21. Martin, J. E.; Herzing, A.; Yan, W.; Li, X.; Koel, B. E.; Kiely, C. J.; Zhang, W.; Langmuir 2008, 24, 4329.

22. Huber, D. L.; Small 2005, 1, 482.

23. Mercado, D. F.; Caregnato, P.; Villata, L. S.; Gonzalez, M. C.; J. Inorg. Organomet. Polym. 2017, in press DOI: 10.1007/ s10904-017-0757-8.

24. Geng, B.; Jin, Z.; Li, T.; Qi, X.; Sci. Total Environ. 2009, 407, 4994. 
25. Mercado, D. F.; Rubert, A.; Magnacca, G.; Malandrino, M.; Sapino, S.; Caregnato, P.; J. Nanosci. Nanotechnol. 2017, 17, 9081.

26. Jang, M.-H.; Lim, M.; Hwang, Y. S.; Environ. Health Toxicol. 2014, 29, e2014022.

27. Yu, T.; Wakuda, K.; Blair, D. L.; Weiss, R. G.; J. Phys. Chem. C 2009, 113, 11546.

28. He, Y.; Zhao, H.; Yao, M.; Weiss, R. G.; J. Polym. Sci., Part A: Polym. Chem. 2017, 55, 3851.

29. Nesher, G.; Marom, G.; Avnir, D.; Eur. J. Inorg. Chem. 2012, 5276.

30. Wang, W.; Zhou, M.; Jin, Z.; Li, T.; J. Hazard. Mater. 2010 , 173,724 .

31. Loos, J.; Sourty, E.; Lu, K.; de With, G.; Bavel, S. V.; Macromolecules 2009, 42, 2581.

32. Pérez, N.; Ruiz-Rubio, L.; Vilas, J. L.; Rodriguez, M.; MartinezMartinez, V.; Leon, L. M.; J. Photochem. Photobiol., A 2016, 315,1 .

33. Cardona, D. M. M.; Wongsa-Ngam, J.; Langdon, T. G.; J. Mater. Res. Technol. 2014, 3, 344.

34. Langford, J. I.; Wilson, A. J. C.; J. Appl. Crystallogr. 1978, 11, 102.

35. Yoo, J. W.; Yun, D. S.; Kim, H. J.; J. Nanosci. Nanotechnol. 2006, 6, 3342 .

36. Vorkapic, D.; Matsoukas, T.; J. Am. Ceram. Soc. 1998, 81, 2815.

37. Jabeen, H.; Chandra, V.; Jung, S.; Lee, J. W.; Kim, K. S.; Kim, S. B.; Nanoscale 2011, 3, 3583.

38. Hanesch, M.; Geophys. J. Int. 2009, 177, 941.

39. Jubb, A. M.; Allen, H. C.; ACS Appl. Mater. Interfaces 2010, 2, 2804 .

40. Rudolph, M.; Erler, J.; Peuker, U. A.; Colloids Surf., A 2012, 397, 16.

41. Geng, B.; Tao, B.; Li, X.; Wei, W.; Nanoscale 2012, 4, 1671.
42. Mercado, D. F.; Magnacca, G.; Malandrino, M.; Rubert, A.; Montoneri, E.; Gonzalez, C.; ACS Appl. Mater. Interfaces 2014, 6, 3937.

43. Liu, Y.; Majetich, S. A.; Tilton, R. D.; Sholl, D. S.; Lowry, G. V.; Environ. Sci. Technol. 2005, 39, 1338.

44. Arnold, W. A.; Roberts, A. L.; Environ. Sci. Technol. 1998, 32, 3017.

45. Giri, S.; Bhaumik, M.; Das, R.; Gupta, V. K.; Maity, A.; Appl. Catal., B 2017, 202, 207.

46. Han, Y.; Yan, W.; Environ. Sci. Technol. 2016, 50, 12992.

47. Li, X.; Elliott, D. W.; Zhang, W.; Crit. Rev. Solid State Mater. Sci. 2006, 31, 111.

48. Hao, L.; Zheng, T.; Jiang, J.; Hu, Q.; Li, X.; Wang, P.; RSC Adv. 2015, 5, 10723.

49. Ponder, S. M.; Darab, J. G.; Environ. Sci. Technol. 2000, 34 , 2564.

50. Liu, H. B.; Chen, T. H.; Chang, D. Y.; Chen, D.; Liu, Y.; He, H. P.; Yuan, P.; Frost, R.; Mater. Chem. Phys. 2012, 133, 205.

51. Gutiérrez, M. C.; López-Mesas, M.; Lacorte, M. T.; Cegarra, J.; Fibers Polym. 2009, 10, 437.

52. Demarger-Andre, S.; Domard, A.; Carbohydr. Polym. 1994, 23 , 211.

53. Jain, S.; Goossens, J. G. P.; Peters, G. W. M.; van Duin, M.; Lemstra, P. J.; Soft Matter 2008, 4, 1848.

54. Ren, J.; Krishnamoorti, R.; Macromolecules 2003, 36, 4443.

55. Kharchenko, S. B.; Douglas, J. F.; Obrzut, J.; Grulke, E. A.; Migler, K. B.; Nat. Mater. 2004, 3, 564.

56. Zarei, M.; Ahmadzadeh, H.; Goharshadi, E. K.; Analyst 2015, $140,4434$.

57. Tuteja, A.; Mackay, M. E.; Hawker, C. J.; Van Horn, B.; Macromolecules 2005, 38, 8000.

Submitted: October 18, 2017 Published online: January 15, 2018 